\title{
Changing perceptions on technological therapy
}

Summarised by Dr. Jessica K Edwards

Educating parents in computer-based therapies for youths with mental health disorders may improve uptake of this therapeutic modality by affected families. Parents who viewed a short presentation on computer-based therapies, reported an improved understanding of the rationale, benefits and helpfulness of this technique, compared to those who viewed an unrelated, control presentation. Specifically, those with low confidence in using technology, demonstrated high gains in knowledge and perceived helpfulness, and parents considered "neurotic or less open" perceived fewer problems with this therapy after viewing the presentation. It remains to be determined whether changes in perception to computer-based therapy, made immediately following an educational presentation, are sustained and boost uptake in children.

Sweeney, G. M., Donovan, C. L., March, S. \& Laurenson, S. D. (2017), Can we improve parent attitudes and intentions to access computer-based therapies for their children and adolescents? Child Adolesc Ment Health, 22:155-162. doi:10.1111/ camh.12180 